\title{
Medidas públicas que favorecen la internacionali- zación de las Pymes. El caso de la Unión Europea
}

Luis Felipe Agramunt

Prof. Titular "Comercio Exterior»

$y$ «Comercialización Internacional».

Facultad de Ciencias Económicas -

Universidad Nacional del Litoral

Email: lagramunt@fce.unl.edu.ar

\section{Marcela Cozzi}

Cientibecaria Facultad de Ciencias Económicas - Universidad Nacional del Litoral

Email:cozzimarcela@gmail.com

\author{
María Fernanda Andrés \\ Prof. Asociada "Comercio Exterior» \\ $y$ «Comercialización Internacional». \\ Facultad de Ciencias Económicas - \\ Universidad Nacional del Litoral \\ Email:mfandres@fce.unl.edu.ar
}

Fecha de recepción

01/03/2014

Fecha de aprobación

$16 / 09 / 2014$

Palabras clave

- Pymes

- internacionalización

- Estado

- dificultades

- competitividad

\section{Resumen}

En este trabajo se ofrece una revisión de la literatura internacional referida al proceso de internacionalización, se identifican los obstáculos que las pequeñas y medianas empresas (Pymes) enfrentan al momento de internacionalizarse y se presenta el concepto de competitividad. El objetivo central consiste en determinar las acciones públicas posibles para mejorar la competitividad empresarial y atenuar los problemas que las Pymes encuentran en el proceso mencionado. En particular, se señalan las medidas que se han tomado en la Unión Europea (UE). Los resultados obtenidos muestran la necesidad de invertir en la internacionalización de las Pymes por parte del sector público. 
Keywords

- SMES

- internationalization

- State

- difficulties

- competitiveness

\section{Abstract}

This paper includes a review of the international literature regarding the internationalization process, identifies the obstacles that Small and Medium Sized Enterprises (SMEs) face when internationalizing and present the concept of competitiveness. The main objective consists in the determination of possible actions to apply in the public sector as a way to improve business competitiveness and tackle SMEs' problems in the process named before. In particular, measures taken by the European Union (EU) are mentioned. The results show the necessity for public investment in SMEs' internationalization.

\section{Introducción}

Actualmente, estamos en presencia de un fenómeno que, si bien dista de ser nuevo, ${ }^{(1)}$ en los últimos tiempos ha adquirido mayor intensidad: se trata de la globalización ${ }^{(2)}$ de los mercados que, a la vez, es causa y consecuencia del fenómeno universal de la internacionalización de las empresas (Canals, 1994).

Como afirma Hessels (2004) la internacionalización es parte de la realidad actual de hacer negocios. Ningún negocio está aislado.

Frente a la globalización, la internacionalización es un proceso cada vez más importante para las pequeñas y medianas empresas (Pymes) alrededor de todo el mundo. Por un lado, el comercio internacional de bienes y servicios lleva a una competencia más feroz en el mercado doméstico de la firma (Tiwari \& Buse, 2007). Por otro lado, les ofrece una cantidad creciente de oportunidades para extender el número de consumidores potenciales más allá de las fronteras nacionales (Onkelinx \& Sleuwaegen, 2008) y para internacionalizar las ventas en nuevos y crecientes mercados, generando ingresos adicionales (Tiwari \& Buse, 2007).

De esta manera, el panorama internacional, una vez considerado como sin límites para las nuevas empresas no sólo es una opción para algunas de ellas, sino también una ruta estratégica para la supervivencia (Oviatt \& McDougall, 2005). Sin importar los motivos, la internacionalización provee a las empresas con oportunidades para mejorar su productividad e incrementar sus chances de supervivencia (Onkelinx \& Sleuwaegen, 2008).

Como sostienen Hessels \& Parker (2013), las Pymes son actores claves en las economías desarrolladas modernas. De acuerdo a datos de la $\mathrm{C}_{0}$ misión Europea (2001a) las Pymes son importantes en la economía europea, alcanzando el $99 \%$ de las empresas, de las cuales el $92 \%$ son micro-empresas, constituyéndose de esa manera en el principal sostén de la economía. Ellas generan más de dos tercios del empleo del sector privado y juegan un rol principal en el crecimiento de la economía; razón 